\title{
Gemeinsame Stellungnahme der Chirurgischen Arbeitsgemeinschaft Coloproktologie (CACP) der Deutschen Gesellschaft für Allgemein- und Viszeralchirurgie (DGAV) und der AG Gastrointestinal- und Abdominaldiagnostik der Deutschen Röntgengesellschaft (DRG) zur Standardisierung der präoperativen Ausbreitungsdiagnostik beim Rektumkarzinom mittels MRT
}

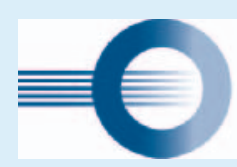

AC Gastrointestinal- und Abdominaldiagnostik

in der Deutschen Röntgengesellschaft

Über viele Jahre galt die neoadjuvante Radiochemotherapie (oder Kurzzeit-Radiotherapie) mit anschließender operativer Resektion als Standardtherapie für Rektumkarzinome des unteren und mittleren Rektumdrittels in den UICC-Stadien II und III (cT3/4 und/oder cN+).
In den letzten Jahren erfolgt eine zunehmend differenzierte Anpassung der Therapieoptionen auf Basis der Tumorinfiltrationstiefe in das mesorektale Fettgewebe, des Tumorabstandes zur mesorektalen Faszie (MRF) sowie des LK-Befalls. So eröffnet die aktuelle S3-Leitlinie die Option einer Therapiedeeskalation. Bei Tumoren mit geringer Infiltrationstiefe (frühes T3 bzw. $\mathrm{T} 3 \mathrm{a} / \mathrm{b})$ und fehlendem LK-Befall kann auf eine vorangehende Strahlentherapie verzichtet und primär operiert werden. In der OCUM-Studie [1] war das Vorgehen einer alleinigen Operation mit risikoadaptierter adjuvanter Chemotherapie auf Basis einer exakten Beschreibung des Abstandes des Tumors zur mesorektalen Faszie (MRF) in vielen Fällen möglich [1]. Weitere Studien (RAPIDO, PRODIGE und OPRA) adressieren wiederum die Intensivierung der adjuvanten Therapie als sog. „totale neoadjuvante 
Therapie“ (TNT), zum Teil (OPRA-Studie) mit intendiertem Organerhalt (,watch and wait“-Konzept) bei lokal fortgeschrittenen Rektumkarzinomen des mittleren und unteren Drittels [2].

Die Stratifizierung der Therapieoptionen beim Rektumkarzinom im Hinblick auf Deeskalation und Eskalation setzt ein adäquates prätherapeutisches Staging voraus. In der Deutschen Neoadjuvansstudie CAO/ ARO/AIO-94 wurden bildgebend als lokal fortgeschritten eingestufte Rektumkarzinome eingeschlossen. Dennoch wurden in der Gruppe der primär operierten Patienten $18 \%$ der Karzinome pathologisch lediglich als T1-2N0 diagnostiziert [3]. In der OCUMStudie wuchs trotz protokollgeführter MRTDiagnostik dieser Anteil auf 27,3\% an [4].

Die Einschätzung der lokalen Tumorausbreitung des Rektumkarzinoms sollte nach aktueller Leitlinie vorzugsweise mittels MRT durchgeführt werden. Gegenüber Ultraschall und $C T$ erlaubt der intrinsische hohe Weichteilkontrast der MRT eine sehr gute Differenzierung der therapierelevanten Risikostrukturen. Die Leitlinie hält allerdings auch kritisch fest, dass die Genauigkeit insbesondere für die MRT wesentlich von der lokalen Expertise abhängt [5].

Die multiparametrische MRT-Diagnostik des Rektumkarzinoms setzt qualitative Standards in technischer Durchführung sowie Auswertung und Befundung voraus. In Zusammenarbeit mit der AG Onkologische Bildgebung, AG Gastrointestinal- und Abdominaldiagnostik und der AG Informationstechnologie sowie klinischen Vertretern u. a. der Deutschen Gesellschaft für Allgemein- und Viszeralchirurgie wurden in einem Konsensus-Meeting 2017 strukturierte Befundvorlagen für das Rektum- und Kolonkarzinom entwickelt und online zur Verfügung gestellt (www.befundung.drg. de). Stellenwert und qualitative Voraussetzungen der MRT für die Therapieplanung beim Rektumkarzinom wurden darüber hinaus als interdisziplinäre Empfehlungen der AIO, ARO, ACO und der deutschen Röntgengesellschaft kürzlich publiziert [6].

Das MRT-Untersuchungsprotokoll enthält obligat hochaufgelöste 2 D (optimal 3 D) -T2-gewichtete Sequenzen über die Tumorregion, die in 2 Raumrichtungen orthogonal bzw. im rechten Winkel zur Achse des betroffenen Rektumabschnittes gekippt werden (sog. paraaxiale und parakoronale Kippung). In gleicher Kippung werden sog. diffusionsgewichtete und kontrastmittelgestützte 3D-GRE-Aufnahmen akquiriert [6-8]. Die diffusionsgewichtete Bildgebung (DWI) erleichtert die Tumordetektion, ist aber für die Beurteilung eines LK-Befalls nur eingeschränkt geeignet. Kontrastmittelgestützte Sequenzen ergänzen die Planung der Strahlentherapie. Bei tiefsitzenden Rektumtumoren im unteren Drittel erfolgt zur Beurteilung des Tumorbezuges zum Sphinkterkomplex die Kippung abweichend hiervon orthogonal zur Achse des Analkanals.

Die Befundung fokussiert auf die therapierelevanten Kriterien. Diese umfassen neben der Tumorausbreitung (T-Kategorie) den lokoregionären und lateralen Lymphknotenbefall (N-Kategorie) [9], den Abstand zur mesorektalen Faszie (MRF) bzw. beim tiefen Rektumkarzinom zum intersphinktären Gewebe als anatomische Fortsetzung der mesorektalen Faszie (mrLowRectalCancerPlane) [8] sowie die Beschreibung einer extramuralen Veneninvasion (EMVI) [10]. Ein mrEMVI-positiver Befund klassifiziert den Tumor für eine neoadjuvante Therapie (ESMO-Guidlines). In den NCCN-Guidelines ist EMVI nicht inkludiert.

Die therapierelevanten Befundungskriterien sollten in strukturierter Form erhoben werden:

1. Tumormetrik: Lokalisation, Abstand zur AC-Linie, Tumorlänge und Tumorabstand zum Oberrand der Puborektalschlinge bzw. zum anorektalen Übergang.

2. Morphologie (konzentrisch, semizirkulär, polypoid) sowie Tumormatrix (solide versus muzinös), Angabe der Uhrzeit.

3. T-Klassifikation mit Subklassifikation T3 in T3a/b und T3c/d $\mathrm{d}^{1}$. Bei Tumoren des

1 Hinweis: In der dritten Auflage des TNMSupplements 2003 für wurde die pathologische Einschätzung die Ramifikation wie folgt angegeben: pT3a $\leq 1 \mathrm{~mm}$, pT3b $\leq 5 \mathrm{~mm}, \mathrm{pT} 3 \mathrm{c}$ $\leq 15 \mathrm{~mm}$ und $\mathrm{pT} 3 \mathrm{~d}>15 \mathrm{~mm}$. In der vierten Auflage von 2013 wurde diese Ramifikation zusammengefasst in: $\mathrm{pT} 3 \mathrm{a} \leq 5 \mathrm{~mm}$ und $\mathrm{pT} 3 \mathrm{~b}$ $>5 \mathrm{~mm}$. Für die klinische Einschätzung in der MRT hat sich diese Zusammenfassung zwar auch durchgesetzt (cT3a/b und cT3c/d), die Notation ist hier aber unverändert geblieben. oberen und mittleren Drittels Lagebezug zur peritonealen Umschlagsfalte (T4a bei Infiltration). Beschreibung der Infiltration anderer Organe (T4b).

4. Bei Tumoren des unteren Drittels Beurteilung des Sphinkterkomplexes mit Unterscheidung Infiltration M. sphincter internus, intersphinktäres Gewebe, M. sphincter externus sowie Levator ani $(\mathrm{T} 4)^{2}$.

5. Lagebezug zur mesorektalen Faszie: Der geringste Abstand zur mesorektalen Faszie wird lokalisiert mit Angabe des Abstandes zur MRF und der Uhrzeit (Tumor selbst, Tumorsatellit oder LKMetastase). Wenn $<1$ mm oder Kontakt/ Infiltration ist der Schnittrand als positiv zu werten, d. h. der potenzielle zirkumferentielle Resektionsrand (CRM) ist positiv.

6. Beurteilung der extramuralen Veneninvasion (EMVI). Die Graduierung erfolgt auf Basis einer 4-teiligen Ordinalskala nach Smith et al. [10].

7. Beurteilung von lokoregionären LK sowie lateralen bzw. extramesorektalen LK.

Die Beurteilung einer prognostisch relevanten LK-Metastasierung stellt bildgebend eine besondere Herausforderung dar. Mikrometastasen können in nicht vergrößerten LK auftreten. Tatsächlich sind ca. $48 \%$ der befallenen LK 5 mm oder kleiner [11]. Dies gilt insbesondere für LK im mesorektalen Fettgewebe knapp ober- und unterhalb $(<1 \mathrm{~cm})$ des Tumorniveaus. Andererseits können LK auch reaktiv vergrößert sein ohne Nachweis einer Metastasierung. Die Beschränkung auf Größenkriterien allein birgt damit das Risiko der Fehlklassifikation. Darüber hinaus ist die LK-Metastasierung abhängig von der Tumorhöhe. Je tiefer der Tumor sitzt, desto wahrscheinlicher ist eine

2 Lt. TNM Supplement 2013 (4. Auflage) wird sowohl die Infiltration des M. sphincter ani internus als auch des M. sphincter ani externus als T4 bewertet. Demgegenüber stehen zum einen die Morphologie (M. sphincter ani internus als Fortsetzung der Tela muscularis propria, M. corrugator ani als Fusion von Längsmuskulatur des Rektums, der parietalen pelvinen Faszie und des M. levator ani (M. pubococcygeus)) und zum anderen die Einschätzung der Low Rectal Cancer Study [8] (intersphinktärer Raum frei = klinisch CRM negativ, intersphinktärer Raum und darüber hinaus infiltriert $=$ CRM positiv). 
LK-Metastasierung in extramesorektale bzw. laterale LK.

Für LK im mesorektalen Fettgewebe sind neben a) ihrer Größe deshalb additiv b) die LK-Form und c) die Signalhomogenität ihrer Binnenstruktur in der sog. T2-Wichtung zu bewerten. Im Hinblick auf die Randbegrenzung kann d) ferner die Randunterbrechung bzw. das Fehlen von sog. „chemical shift“-Artefakten in T2-gewichteten Bildern als Kriterium für eine Tumorinfiltration gewertet werden. Die Genauigkeit der MRT im LK-Staging kann durch die Kombination dieser Kriterien auf bis zu 78-85\% gesteigert werden [12].

Ein positiver LK-Befall stuft Patienten a priori und unabhängig von der T-Kategorie (T1-T3a/b) in eine intermediäre Risikogruppe ein. Aus Behandlungssicht ist die prätherapeutische Beurteilung des Lymphknotenstatus ein kritisches Moment. Einerseits besteht eine große diagnostische Unsicherheit, andererseits sind daran relevante therapeutische Konsequenzen gebunden, da sowohl die aktuellen deutschen Leitlinien als auch die NCCN für einen klinisch lymphknotenpositiven Tumor eine neoadjuvante Therapie vorsehen. Lediglich die ESMOLeitlinie erlaubt für definierte Fälle $(\leq \mathrm{T} 3 \mathrm{a} / \mathrm{b}$, CRM-negativ, EMVI-negativ) die primäre OP bei LK-Positivität. Die deutsche S3-Leitlinie nimmt zu dieser Problematik wie folgt Stellung:

„Als Problem jeder neoadjuvanten Therapie muß das potentielle „Overstaging " und die daraus resultierende „Überbehandlung“ von Patienten gewertet werden, bei denen fälschlicherweise ein lymphknotenpositiver Tumor $(\mathrm{cN}+)$ diagnostiziert wurde.

Bei der Beurteilung der Lymphknoten sind Sensitivität (55-73\%) und Spezifität (74-78\%) aller Verfahren derzeit unbefriedigend. Dies ist u. a. dadurch bedingt, dass einerseits eine reaktive Lymphknotenvergrößerung auftritt, andererseits auch Lymphknoten von $5 \mathrm{~mm}$ und kleiner Metastasen enthalten können. Aus diesem Grund sollte die Indikation zu einer neoadjuvanten Therapie sehr zurückhaltend gestellt werden, wenn sie allein auf der Beschreibung suspekter Lymphknoten in der prätherapeutischen Bildgebung basiert.“
Zusammenfassend wird daher vorgeschlagen, die bildmorphologische Beurteilung von Lymphknoten in der T2-Wichtung systematisch nach Größe, Form, Binnenstruktur und Randbegrenzung vorzunehmen sowie abschließend die Befundsicherheit nach einer 3-teiligen Konfidenzskala vorzunehmen: 1. eindeutiger Tumorbefall ( $\mathrm{N}$ plus), 2. fraglicher Tumorbefall ( $\mathrm{Nx}$ ) und 3. sicher kein Tumorbefall (NO).

Die o.g. Arbeitsgruppen werden in Zusammenarbeit und in Ergänzung zu den hier erwähnten Kriterien weitere Vorschläge und Präzisierungen erarbeiten, die den jeweiligen Fachgesellschaften zur Konsentierung vorgelegt werden.

Sigmar Stelzner, Stefan Fichtner-Feigl, Volker Kahlke, Anton Kroesen, Peter Kienle für die Chirurgische Arbeitsgemeinschaft Coloproktologie (CACP) der Deutschen Gesellschaft für Allgemein- und Viszeralchirurgie (DGAV)

Johannes Weßling, Lars Grenacher

für die Arbeitsgemeinschaft Gastrointestinal- und Abdominaldiagnostik der Deutschen Röntgengesellschaft (DRG)

\section{Korrespondenzadresse:}

Priv.-Doz. Dr. med. Sigmar Stelzner

Klinik für Allgemein- und Viszeralchirurgie

Städtisches Klinikum Dresden-Friedrichstadt

Friedrichstr. 41

D-01067 Dresden

E-Mail: sigmar.stelzner@klinikum-dresden.de

Erstpubliziert in S. Stelzner, S. FichtnerFeigl, V. Kahlke, P. Kienle, A. Kroesen, L. Grenacher, J. Wessling (2021) Standardisierung der präoperativen Ausbreitungsdiagnostik beim Rektumkarzinom mittels MRT. Der Chirurg 5. doi:10.1007/s00104-021-01411-y

\section{Literatur}

[1] Ruppert R, Junginger T, Ptok $\mathrm{H}$ et al. Oncological outcome after MRI-based selection for neoadjuvant chemoradiotherapy in the OCUM Rectal Cancer Trial. Br J Surg 2018; 105: 1519 1529
[2] Konsentierte Stellungnahme der AIO, der ACO und der ARO zur neoadjuvanten Therapie beim Rektumkarzinom 2020. https://www. aio-portal.de/tl_files/aio/stellungnahmen/ Stellungnahme\%20AIO_ACO_ARO\%20 Rektumkarzinom\%20_\%2007_2020.pdf; aufgerufen am 12.10.2020

[3] Sauer R, Becker H, Hohenberger W et al. Preoperative versus postoperative chemoradiotherapy for rectal cancer. N Engl I Med 2004; 351: $1731-1740$

[4] Ruppert R, Kube R, Strassburg J et al. Avoidance of Overtreatment of Rectal Cancer by Selective Chemoradiotherapy: Results of the Optimized Surgery and MRI-Based Multimodal Therapy Trial. J Am Coll Surg 2020; 231: 413425

[5] Leitlinienprogramm Onkologie (2019) S3Leitlinie Kolorektales Karzinom, Version 2.1. http://www.leitlinienprogramm-onkologie. de/leitlinen/kolorektales-karzinom/; aufgerufen 17.07.2020

[6] Attenberger UL, Clasen S, Ghadimi M et al. Importance and Qualitative Requirements of Magnetic Resonance Imaging for Therapy Planning in Rectal Cancer - Interdisciplinary Recommendations of AIO, ARO, ACO and the Germany Radiological Society. Fortschr Röntgenstr 2021 [in press]

[7] Brown G, Daniels IR, Richardson C et al. Techniques and trouble-shooting in high spatial resolution thin slice MRI for rectal cancer. $\mathrm{Br}$ ] Radiol 2005; 78: 245-251

[8] Battersby N], How P, Moran B et al. Prospective Validation of a Low Rectal Cancer Magnetic Resonance Imaging Staging System and Development of a Local Recurrence Risk Stratification Model: The MERCURY II Study. Ann Surg 2016; 263: 751-760

[9] Brown G, Richards C], Bourne MW et al. Morphologic predictors of lymph node status in rectal cancer with use of highspatial-resolution MR imaging with histopathologic comparison. Radiology 2003; 227: 371

[10] Smith NY, Barbachano Y, Norman AR et al. Prognostic significance of magnetic resonance imaging-detected extramural vascular invasion in rectal cancer. Br J Surg 2008; 95 : 229-236

[11] Steup W, Moriya Y, Van der Velde C et al. Patterns of lymphatic spread in rectal cancer. A topographical analysis on lymph node metastases. Eur J Cancer 2002; 38: 911-918

[12] Nougaret S, Reinhod C, Mikhael HW et al. The use of MR imaging in the treatment planning for patients with rectal carcinoma: have you checked the "DISTANCE"? Radiology 2013 268: $330-344$ 